\title{
Monitoring Land Cover Change Using Remote Sensing (RS) and Geographical Information System (GIS): A Case of Golden Pride and Geita Gold Mines, Tanzania
}

\author{
Caren Kahangwa ${ }^{1 *}$, Cuthbert Nahonyo ${ }^{1}$, George Sangu ${ }^{2}$ \\ ${ }^{1}$ Department of Zoology and Wildlife Conservation, University of Dar es Salaam, Dar es Salaam, Tanzania \\ ${ }^{2}$ Department of Botany, University of Dar es Salaam, Dar es Salaam, Tanzania \\ Email: *Caren.kahangwa@gmail.com
}

How to cite this paper: Kahangwa, C., Nahonyo, C. and Sangu, G. (2020) Monitoring Land Cover Change Using Remote Sensing (RS) and Geographical Information System (GIS): A Case of Golden Pride and Geita Gold Mines, Tanzania. Journal of Geographic Information System, 12, 387-410. https://doi.org/10.4236/jgis.2020.125024

Received: August 3, 2020

Accepted: September 7, 2020

Published: September 10, 2020

Copyright $\odot 2020$ by author(s) and Scientific Research Publishing Inc. This work is licensed under the Creative Commons Attribution International License (CC BY 4.0).

http://creativecommons.org/licenses/by/4.0/

(c) (i) Open Access

\begin{abstract}
This study monitored land cover change in the mining sites of Golden Pride Gold Mine (GPGM) and Geita Gold Mine (GGM), Tanzania. The satellite data for land cover classification for the years 1997, 2010 and 2017 were obtained from the United States Geologic Survey Departments (USGS) online database and were analyzed using Arc GIS 10 software. Supervised classification composed of seven classes namely forest, bushland, agriculture, water, bare soil, urban area and grassland, was designed for this study, in order to classify Landsat images into thematic maps. In addition, future land cover changes for the year 2027 were simulated using a Cellular Automata (CA)Markov model after validating the model using the Land Cover for the year 2017. The results from the LULC analysis showed that forest was the most dominant land cover type in 1997 at GPGM and GGM covering 510 ha (52.1\%) and 9833 ha (49.7\%) respectively. In 2017, the forest area decreased and the bushland replaced forest to be the most dominant land cover type covering 219 ha (22.4\%) for GPGM and 8878 ha (44.9\%) for GGM. Based on the CA-Markov model, a predicted land cover map for 2027 was dominated by forest covering 340 ha (34.7\%) and 8639 ha (43.7\%) for GPGM and GGM respectively. An overall accuracy and kappa coefficient for GPGM were $74.7 \%$ and $70.2 \%$ respectively and for GGM were $71.4 \%$ and $66.1 \%$ respectively. Thus, land cover changes resulting from mining activities involve reduction of forest land hence endangers biodiversity. GIS and remote sensing technologies are potential to detect the trend of changes and predict future land cover. The findings are crucial as it provides basis for land use planning and intensifies monitoring programs in the mining areas of
\end{abstract}


Tanzania.

\section{Keywords}

Land Cover, Remote Sensing, Change Detection, Accuracy Assessment

\section{Introduction}

Mining is an extraction of valuable materials from the earth's surface usually from an ore which consequently results in loss of vegetation, loss of soil and loss of rocks [1]. Mining regions are geologically defined by economically feasible mineral resources and they often undergo abrupt and extensive changes in the land cover [2]. Large-scale mining is one of the main growing industries in Tanzania. Large-scale mining especially open-pit involves the clearing of large area of land and forest resulting in serious land cover changes [3]. During the past two decades, Golden Pride Gold Mine (GPGM) and Geita Gold Mine (GGM) among the gold mines within Lake Victoria Gold field have undergone significant land cover changes due to mining operations within the licensed mining areas. Land cover is the biotic or abiotic features that cover the earth surface such as forest, bare soil, grassland, bushland and water [3].

From environmental point of view, the dynamic process of land cover change has been described as the most remarkable anthropogenic disruption of the environment [4] [5]. It is a dynamic variable because it reflects the interaction between socio-economic activities and regional environmental changes, and for this reason, it is necessary to be updated frequently [6]. Land Cover is necessary in identifying and mapping natural resources and human activities which have a great effect on landscape and soil degradation [7] [8]. Information on land cover plays a key role in natural resources management and spatial planning [9] [10].

This study used Remote Sensing (RS) and Geographical Information System (GIS) to monitor the trend of land cover change in the mining areas and to predict the future land cover [11]. Remote sensing techniques have been used over the years and have proven to be of great value for monitoring changes at regular intervals [8]. Using satellite remote sensing data is a practical option to identify and map the land cover categories [12]. Land cover mapping using satellite remote sensing images has become widely popular in the last decades [13]. The reasons for selecting satellite image periods for this study considered the phases of mining projects from pre-mining, during active operations and post-mining phases. In addition, GIS tools are used to create the geo-database and integrate data extracted from satellite images with classes from the currently available land cover models [6]. However, developing countries still lag behind significantly compared with the developed countries in the application of remote sensing and GIS technologies. Land cover change models are very helpful for evaluating anthropogenic and natural impacts, predicting future patterns of land use changes, 
aiding stakeholders of natural resources to achieve sustainable environments and planning and management of land transformation [11]. A GIS can be integrated to predict the future scenario of land cover using Cellular Automata (CA) Markov model.

CA Markov model is widely used to predict and analyze likely future trajectory of LULC within a specified period and to characterize the dynamics of land use/cover, forest cover, coastal management, wetland landscape and so forth [14] [15] [16] [17]. The heart of a CA Markov model is the transition matrix between different temporal images to predict the future events [18] [19]. In general, various traditional modellings in geography create a spatial layout of present and past land surfaces using dynamic optimization techniques [18]. Commonly used models for estimating land cover changes are statistical models, analytical equation-based models, evolutionary models, cellular models, Markov models, hybrid models, expert system models and multi-agent models [20]. Currently, the most commonly used models in land cover change monitoring and prediction are the mixed model [20]. The CA-Markov model is the mixture of the Cellular Automata and Markov models. CA Markov model has many advantages for geographic modelling; it is capable of supporting very large parameter spaces for simulation, the model makes an implicit use of space and spatial complexity by simple local raster transformation, it is capable of combining space-time relationships, as well as supporting separate notions of space and time. Moreover, the model is capable of representing pattern, process, form and function. CA Markov model is vital in helping land use planners to make land use planning decision.

Tanzania, like many other developing countries, the large-scale gold mining activities typically decrease land covered by vegetation through the process of forest clearing for formation of mining components such as pits, Waste Rock Dumps and Tailing Storage Facilities. However, the countries are still using traditional field investigation method instead of remote sensing and GIS technologies to monitor the trend of land cover changes in the mining areas. This traditional technology is low efficient, high labor demanding and impossible to conduct continuously, especially in large scale areas. Therefore, the use of remote sensing (RS) in combination with Geographical Information System (GIS) has proved to be effective in the spatial and temporal monitoring of the land cover changes in the mining areas.

Numerous researches have been published on the effects of large scale mining activities on land cover worldwide [2] [3] [11] [21] [22] [23]. In general, studies revealed that mining activities cause extensive land use changes, which pose significant management challenges for mining companies and regulatory authorities. The effects identified in the studies include land degradation and conversion of land from forest covers to non-forest covers such as built-up areas, bare lands, Waste Rock Dumps and abandoned quarries filled with water.

In Tanzania, [24] monitored geomorphic and hydrologic change at GGM us- 
ing Digital Elevation Model (DEM) data from the Space shuttle Radar Topography Mission (SRTM) and the Advanced Spaceborne Thermal Emission and Reflection Radiometer (ASTER). The authors assessed the effects of mining activities on farmers, village water supplies, and community forests using a hydrologic flow model. The results indicated that more than 81 million $\mathrm{m}^{3}$ of waste has been deposited in the tailings storage facility and waste rock dumps. [1] also analyzed the impact of mining activities on land cover using Arc View GIS and ERDAS Imagine. The results indicated that there was significant conversion of forest cover into non-forest cover from 1991-2011 in Nanyala Ward. The author concluded that as the mining activities increase, the area affected by mining increases posing a serious threat to the environments. From the above background, it is evidently that, as far as land cover change due to gold mining operation is concerned, there is limited knowledge evaluating and predicting the future land covers in the gold mining environment of Tanzania. The aim of this study therefore, was to monitor land cover changes from 1997 to 2017 and then predict the future land cover for the year 2027 at GPGM and GGM mining licensed areas. The prediction of future land cover is important in the sense that, it will enhance understanding of the present land cover and will provide some foundation for effective planning of the future land use. This will help in monitoring the mining landscape for sustainable development to meet the needs of the present and future generation. We recommend effective management of degraded mining areas by intensifying progressive rehabilitation programs through planting trees as this will help to achieve positive post mine closure outcomes.

\section{Materials and Methods}

\subsection{Overview of the Study Area}

GPGM and GGM are located in the Sukuma land Greenstone belt within Lake Victoria Goldfield in Northern Tanzania. Lake Victoria Goldfield is among the three principle goldfields which are the main producers of gold in Tanzania. Other goldfields are Mpanda and Lupa located in the Western and Southern Tanzania respectively [25]. Geographically, the GPGM is located at latitude: 040 23'31"S and longitude 0320 53'55"E in Nzega District, Tabora Region, Tanzania. It is approximately $18 \mathrm{~km}$ north of Nzega Township, and $200 \mathrm{~km}$ south of Mwanza regional center. Average annual rainfall is between $700-800 \mathrm{~mm}$ per year and average temperatures range from $22^{\circ} \mathrm{C}$ to $27^{\circ} \mathrm{C}$ [26]. Whereas, GGM is located at latitude: $02052^{\prime} 03^{\prime \prime S}$ and longitude: $032011^{\prime} 11^{\prime \prime} \mathrm{E}$ it is approximately 4 $\mathrm{km}$ west of Geita town and $90 \mathrm{~km}$ south-west of Mwanza City in north-western Tanzania (Figure 1). There is very little annual variation in daytime temperatures throughout the year with an annual average daily temperature of $25^{\circ} \mathrm{C}$ as recorded at GGM. Evaporation rates throughout the year are related to the monthly rainfall and are much greater during the dry season, yet are overall relatively low, ranging between 70 and $140 \mathrm{~mm}$ per month. 


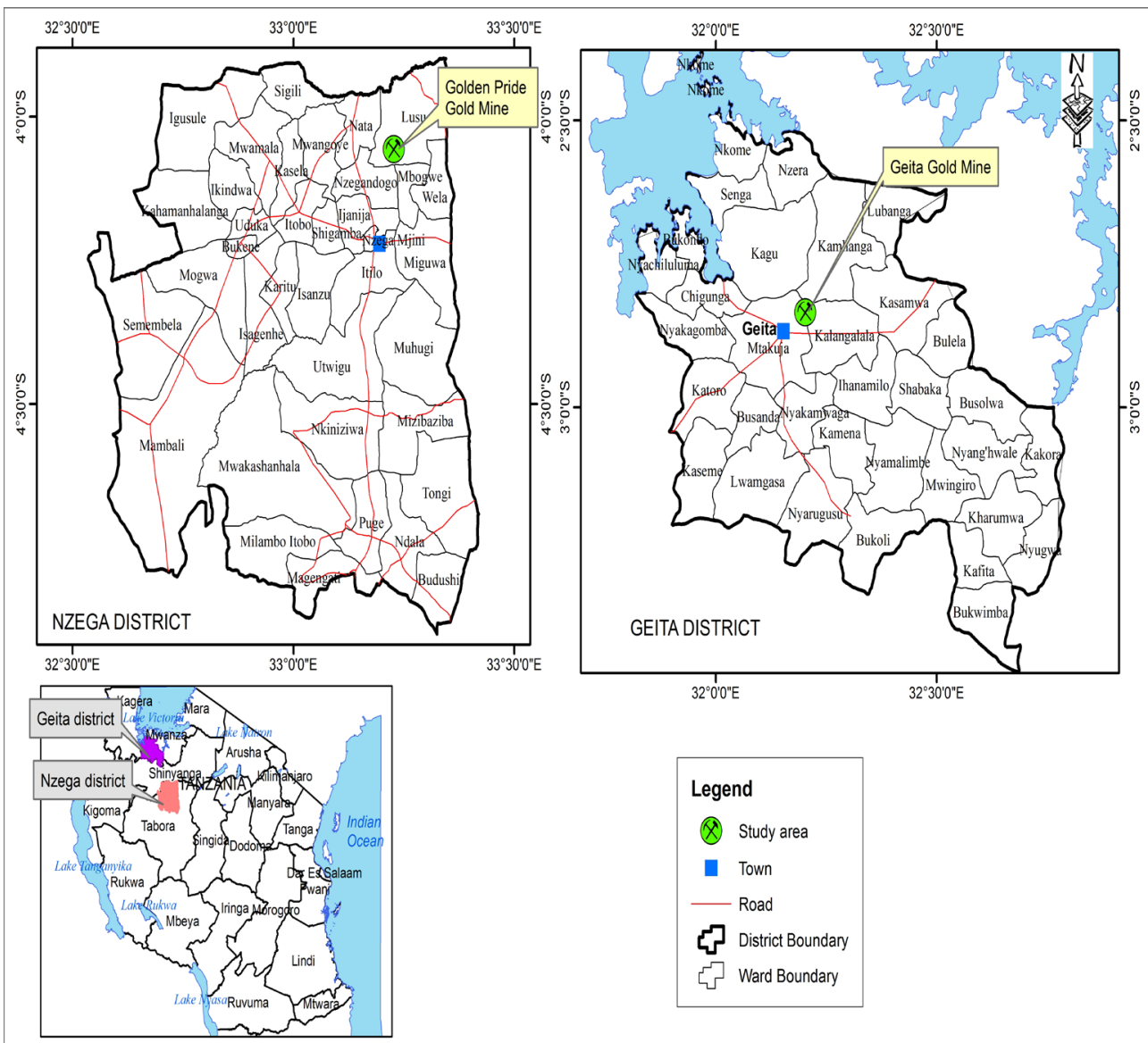

Figure 1. Location map of golden pride gold mine and Geita gold mine.

\subsection{Research Materials}

This paper covers Land Cover classification and Accuracy Assessment. In order to determine the effects of large-scale mining on the land cover of the study area, land cover satellite data sets for the years 1997 and 2010 were delivered from Landsat Thematic Mapper and for the year 2017 were delivered from Landsat 8 Operational Land Imager (OLI). The satellite data sets were obtained from the United States Geologic Survey Department (USGS) online database through http//earthexplorer.gov. Various digital image processing techniques were employed to prepare land cover maps of the study areas.

\subsection{Research Methods}

\subsubsection{Remote Sensing Data Collection}

To prepare the base maps, the Landsat satellite images (1997, 2010 and 2017) were collected from the official website of US Geological Survey (USGS). Landsat Path 171 row 62 covered GGM and Landsat Path 170 row 63 covered GPGM. The pixel size of the images was $30 \times 30 \mathrm{~m}$. Accordingly, the land cover was classified into seven classes namely forest, bushland, agriculture, water, bare soil, urban area and grassland, for the three sampling periods i.e. 1997, 2010 and 2017. 


\subsubsection{Data Acquisition and Processing}

Time analyses of land cover change require a proper selection and preparation to ensure the compatibility of the Landsat images. In this study, the images were selected from the same season (July-September) and with minimal cloud cover $(<10 \%)$. The images were from different sensors with similar spectral resolution i.e. 30 metres.

\subsubsection{Land Cover Change Detection}

The land cover change detection was done using ArcGIS10.3 software. The two classified Land cover layers i.e. Land cover 1997 and 2010 and Land cover 2010 and 2017 were used. Spatial analysis was done using the zonal tabulate area function and was used to generate land cover change matrix. The function calculates cross-tabulated areas between two datasets and provides output of a table that displays a record for each unique value of the zone dataset and a field for each unique value of the class dataset. Also, the land cover change analyses were performed and cross tabulation was done using Spatial analysis function in ArcGIS 10 software [5].

\subsubsection{Land Cover Change Modelling}

Coupled Cellular Automata (CA)-Markov model was employed to conduct Land cover change modelling. The CA-Markov model combination represents advancement in spatio-temporal dynamic modelling and forecasting, and enable achieving a better simulation of land cover changes both in quantity and space based on studies of land use changes in the past [14] [20]. The algorithms in the IDRIS Andes package integrate the functions of the CA filter and Markov process, using conversion tables and conditional probabilities from the conversion map applied to simulate and forecast the states of Land cover change [15]. Therefore, to simulate future land cover changes for this study sites using a CA-Markov model, the following specific processes were followed: 1) After converting the vector data to raster, the classified land cover maps for the years 1997, 2010 and 2017 were used to obtain the transition matrices for the land cover categories between 1997 and 2010 as well as 2010 and 2017 based on the first-order Markov model. 2) The transition suitability maps, which were used to predict the land cover in 2017 and to simulate the distribution in 2027 were generated based on the main transitions that occurred among the land cover categories from 2010 to 2017. In addition, to determine CA filters, the regular 5 $\times 5$ contiguity filter was used as the neighbourhood definition. 3) Based on the CA-Markov model approach, the land cover for the year 2017 was modelled using the transition probabilities from 1997 to 2010 with the land cover base map from the year 2010. Kappa statistics were used to assess the accuracy of the forecasted 2017 land cover map to evaluate its agreement with the actual 2017 land cover map. 4) Finally, following the same process, the land cover for the year 2027 was projected with the CA-Markov model in IDRISI using the transition probabilities from 2010 to 2017 and the land cover base map from the year 2017. 


\subsubsection{Cross Tabulation}

Idrisi software was used to perform a cross-tabulation analysis. It compared images containing categorical variables of two types. Cross Tabulation provides the information on the frequencies with which each land cover classes remained either unchanged or has changed to one of the other classes, using two thematic maps of different dates [27]. Three cross-tabulation tables were generated from thematic maps 1997-2010, 2010-2017 and 2017-2027.

\subsubsection{Accuracy Assessment}

The process of determining accuracy for a classified image is one of the most important steps undertaken in post-classification [1]. To acquire a better accuracy, ground truthing, or physical appearance in the study site is necessary [28]. However, in these cases, training points located in remote areas could not be visited in the field due to time and cost constraints. Kappa statistics was used to assess the accuracy of the forecasted land cover map to evaluate its agreement with the actual land cover map and the analysis was done using validate tool in Idris Selva software. The classified land cover for year 2017 was compared using projected land cover for year 2017.Kappa coefficient estimates the agreement between map and reality and it ranges from 0 to 1 , where 0 represent total disagreement and 1 total agreement [6]. [29] also provides the interpretation of Kappa statistics as presented in Table 1.

\section{Results}

\subsection{Land Cover Classification}

The Landsat images for the study area were classified in order to identify the changes in the cover between the four periods, i.e. 1997, 2010, 2017 and 2027 and this yielded four Land Cover maps from the satellite images for each site. Seven major land cover categories: agriculture, bare soil, built-up area/urban area, bush land, forest, grassland and water were recorded in Golden Pride and Geita Gold Mines respectively. The land cover maps of the study areas for different periods (1997, 2010, 2017 and 2027) are presented in Figure 2 for GPGM and Figure 3 for GGM. Graphical presentation of the areas for each land cover category for the years 1997, 2010 and 2017 are shown in Figure 4 for GPGM and Figure 5 for GGM. The results on land cover and the extent of change in hectares and percentage of each land cover category for the three years were determined as presented in Table 2 and Table 3 for GPGM and GGM respectively. Transition matrices are presented on Tables 4-6 for GPGM and Tables 7-9 for GGM. The land cover categories delineated in the study areas reveal the changes in land cover (in hectares and percentages) that have taken place during the period between 1997-2017 as being due to the expansion of mining activities. Classified Accuracy Assessment report and Kappa statistics for GPGM are provided in Table 10 and Table 11 respectively and for GGM are presented on Table 12 and Table 13 respectively. 


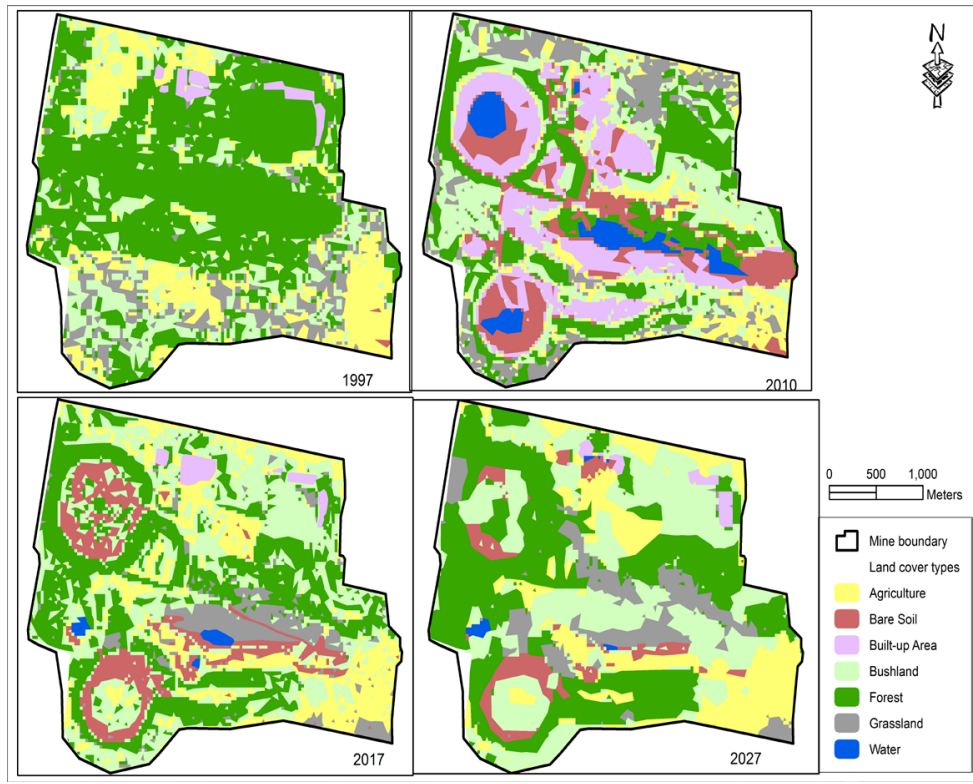

Figure 2. Land cover map at GPGM.
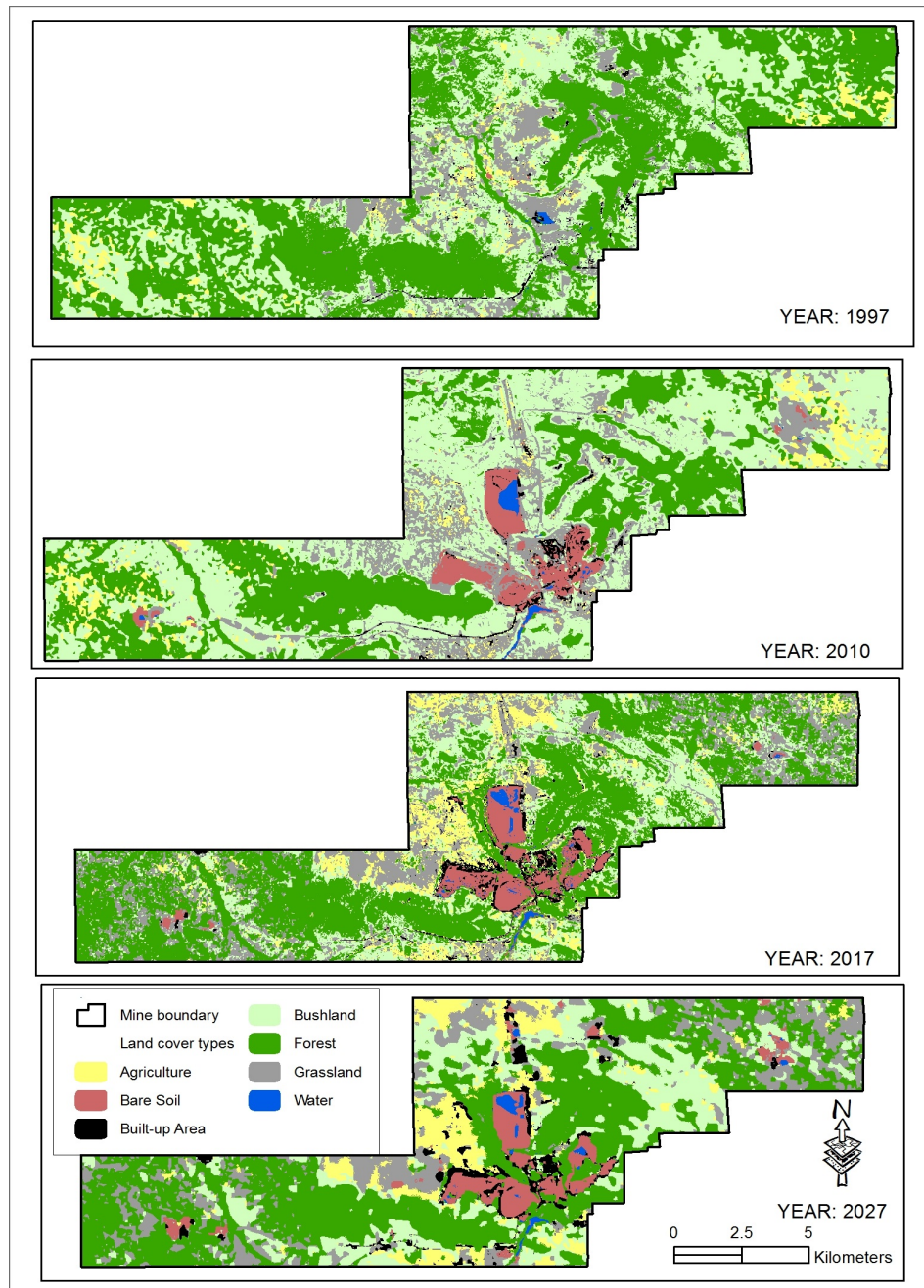

Figure 3. Land cover map at GGM. 


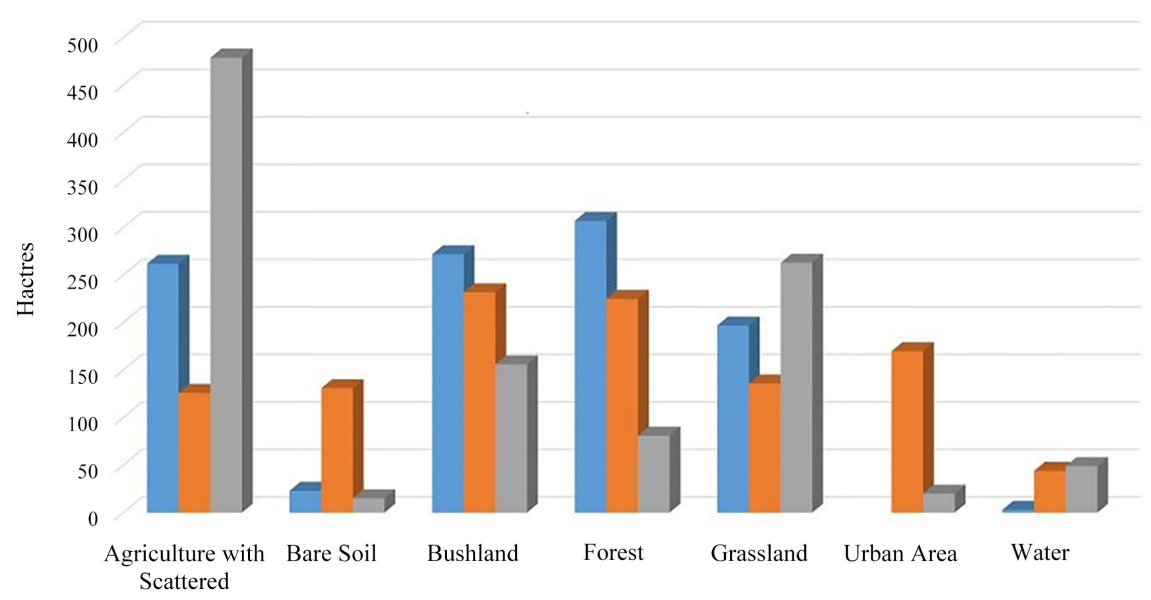

Figure 4. Total area in hectares of each land cover category at GPGM.

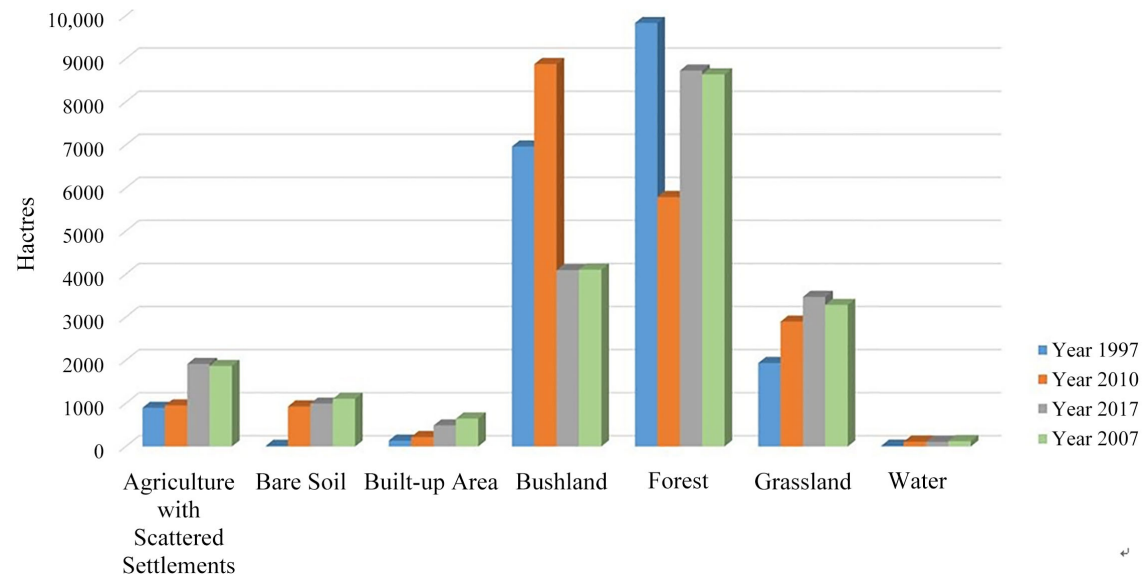

Figure 5. Total area in hectares of each land cover category at GGM.

Table 1. Interpretation of Kappa statistics [29].

\begin{tabular}{|c|c|}
\hline \multirow{2}{*}{ Kappa } & Interpretation of Kappa \\
\hline & Agreement \\
\hline$<0$ & Less than chance agreement \\
\hline $0.01-0.20$ & Slight agreement \\
\hline $0.21-0.40$ & Fair agreement \\
\hline $0.41-0.60$ & Moderate agreement \\
\hline $0.61-0.80$ & Substantial agreement \\
\hline $0.81-0.99$ & Almost perfect agreement \\
\hline
\end{tabular}

Table 2. GPGM land cover change for the years 1997-2017 and prediction for 2027.

\begin{tabular}{|c|c|c|c|c|c|c|c|c|c|c|c|}
\hline \multirow{3}{*}{ Land Use/Cover Types } & \multicolumn{8}{|c|}{ Land Cover } & \multicolumn{3}{|c|}{ Land cover change } \\
\hline & \multicolumn{2}{|c|}{ Year: 1997} & \multicolumn{2}{|c|}{ Year: 2010} & \multicolumn{2}{|c|}{ Year: 2017} & \multicolumn{2}{|c|}{ Year: 2027} & \multirow{2}{*}{$\frac{1997-2010}{\mathrm{Ha}}$} & \multirow{2}{*}{$\frac{2010-2017}{\mathrm{Ha}}$} & \multirow{2}{*}{$\frac{2017-2027}{\mathrm{Ha}}$} \\
\hline & $\mathrm{Ha}$ & $\%$ & $\mathrm{Ha}$ & $\%$ & $\mathrm{Ha}$ & $\%$ & $\mathrm{Ha}$ & $\%$ & & & \\
\hline $\begin{array}{c}\text { Agriculture with } \\
\text { Scattered Settlements }\end{array}$ & 176.0 & 18.0 & 100.0 & 10.2 & 173.0 & 17.7 & 177.0 & 18.1 & -76.0 & 73.0 & 4.0 \\
\hline
\end{tabular}


Continued

\begin{tabular}{ccccccccccccc}
\hline Bare Soil & 1.0 & 0.1 & 129.0 & 13.2 & 98.0 & 10.0 & 52.0 & 5.3 & 128.0 & -31.0 & -46.0 \\
Bushland & 187.0 & 19.1 & 219.0 & 22.4 & 308.0 & 31.5 & 299.0 & 30.5 & 32.0 & 89.0 & -9.0 \\
Forest & 510.0 & 52.1 & 209.0 & 21.3 & 303.0 & 30.9 & 340.0 & 34.7 & -301.0 & 94.0 & 37.0 \\
Grassland & 87.0 & 8.9 & 109.0 & 11.1 & 73.0 & 7.5 & 94.0 & 9.6 & 22.0 & -36.0 & 21.0 \\
Built up Area & 18.0 & 1.8 & 170.0 & 17.4 & 17.0 & 1.7 & 12.0 & 1.2 & 152.0 & -153.0 & -5.0 \\
Water & 0.0 & 0.0 & 44.0 & 4.5 & 7.0 & 0.7 & 4.0 & 0.4 & 44.0 & -37.0 & -3.0 \\
Total & $\mathbf{9 7 9 . 0}$ & $\mathbf{1 0 0 . 0}$ & $\mathbf{9 8 0 . 0}$ & $\mathbf{1 0 0 . 0}$ & $\mathbf{9 7 9 . 0}$ & $\mathbf{1 0 0 . 0}$ & $\mathbf{9 7 8 . 0}$ & $\mathbf{1 0 0 . 0}$ & & & \\
\hline
\end{tabular}

Table 3. GGM land cover change for the years 1997-2017 and prediction for 2027.

\begin{tabular}{|c|c|c|c|c|c|c|c|c|c|c|c|}
\hline \multirow{3}{*}{ Land Use/Cover Types } & \multicolumn{6}{|c|}{ Land Cover } & \multicolumn{5}{|c|}{ Land cover change } \\
\hline & \multicolumn{2}{|c|}{ Year: 1997} & \multicolumn{2}{|c|}{ Year: 2010} & \multicolumn{2}{|c|}{ Year: 2017} & \multicolumn{2}{|c|}{ Year: 2027} & \multirow{2}{*}{$\frac{1997-2000}{\mathrm{Ha}}$} & \multirow{2}{*}{$\frac{2010-2017}{\mathrm{Ha}}$} & \multirow{2}{*}{$\frac{2017-2027}{\mathrm{Ha}}$} \\
\hline & $\mathrm{Ha}$ & $\%$ & $\mathrm{Ha}$ & $\%$ & $\mathrm{Ha}$ & $\%$ & $\mathrm{Ha}$ & $\%$ & & & \\
\hline $\begin{array}{c}\text { Agriculture with } \\
\text { Scattered Settlements }\end{array}$ & 899.0 & 4.5 & 953.0 & 4.8 & 1917.0 & 9.7 & 1869.0 & 9.4 & 54.0 & 964.0 & -48.0 \\
\hline Bare Soil & 14.0 & 0.1 & 930.0 & 4.7 & 996.0 & 5.0 & 1107.0 & 5.6 & 916.0 & 66.0 & 111.0 \\
\hline Built-up Area & 137.0 & 0.7 & 219.0 & 1.1 & 488.0 & 2.5 & 648.0 & 3.3 & 82.0 & 269.0 & 160.0 \\
\hline Bushland & 6964.0 & 35.2 & 8878.0 & 44.9 & 4093.0 & 20.7 & 4107.0 & 20.8 & 1914.0 & -4785.0 & 14.0 \\
\hline Forest & 9833.0 & 49.7 & 5792.0 & 29.3 & 8728.0 & 44.1 & 8639.0 & 43.7 & -4041.0 & 2936.0 & -89.0 \\
\hline Grassland & 1916.0 & 9.7 & 2898.0 & 14.6 & 3453.0 & 17.5 & 3286.0 & 16.6 & 982.0 & 555.0 & -167.0 \\
\hline Water & 19.0 & 0.1 & 111.0 & 0.6 & 108.0 & 0.5 & 126.0 & 0.6 & 92.0 & -3.0 & 18.0 \\
\hline Total & $19,782.0$ & 100.0 & $19,781.0$ & 100.0 & $19,783.0$ & 100.0 & $19,782.0$ & 100.0 & & & \\
\hline
\end{tabular}

Table 4. Land cover change by cross tabulation for GPGM for the years 1997-2010.

\begin{tabular}{|c|c|c|c|c|c|c|c|c|}
\hline \multirow[b]{2}{*}{ Year 2010} & \multicolumn{7}{|c|}{ Year: 1997} & \multirow[b]{2}{*}{$\begin{array}{c}\text { Gross gain } \\
\text { [Total-unchanged] }\end{array}$} \\
\hline & $\begin{array}{c}\text { Agriculture with } \\
\text { scattered settlements }\end{array}$ & Bare Soil & Built-up & Bushland & Forest & Grassland & $\begin{array}{l}\text { Total in } \\
\text { year } 2010\end{array}$ & \\
\hline $\begin{array}{c}\text { Agriculture with } \\
\text { scattered settlements }\end{array}$ & 30.8 & 0.7 & 1.2 & 14.4 & 40.9 & 11.6 & 99.5 & 68.8 \\
\hline Bare Soil & 19.7 & 0.2 & 1.4 & 22.4 & 70.7 & 13.5 & 127.8 & 127.6 \\
\hline Built-up Area & 24.8 & 0 & 6.3 & 32.5 & 96.2 & 13 & 172.7 & 166.4 \\
\hline Bushland & 37.4 & 0 & 4.5 & 45.8 & 112.1 & 17.8 & 217.7 & 171.9 \\
\hline Forest & 29.2 & 0 & 3.7 & 38.1 & 119.8 & 19.7 & 210.4 & 90.6 \\
\hline Grassland & 26 & 0 & 0.5 & 19.4 & 53.4 & 7.8 & 107.1 & 99.3 \\
\hline Water & 6.9 & 0 & 0.5 & 13.9 & 18.8 & 3.3 & 43.5 & 43 \\
\hline Total in year 1997 & 174.8 & 0.9 & 18 & 186.5 & 511.8 & 86.8 & 978.8 & \\
\hline Gross loss & 144 & 0.7 & 11.7 & 140.7 & 392 & 78.9 & & \\
\hline
\end{tabular}


Table 5. Land cover change by cross tabulation for GPGM for the years 2010-2017.

\begin{tabular}{|c|c|c|c|c|c|c|c|c|c|}
\hline \multicolumn{10}{|c|}{ Year: 2010} \\
\hline Year 2017 & $\begin{array}{c}\text { Agriculture with } \\
\text { scattered settlements }\end{array}$ & $\begin{array}{c}\text { Bare } \\
\text { Soil }\end{array}$ & $\begin{array}{l}\text { Built-up } \\
\text { Area }\end{array}$ & Bushland & Forest & Grassland & Water & $\begin{array}{l}\text { Total in } \\
\text { year } 2017\end{array}$ & $\begin{array}{c}\text { Gross gain } \\
\text { [Total-unchanged] }\end{array}$ \\
\hline $\begin{array}{c}\text { Agriculture with } \\
\text { scattered settlements }\end{array}$ & 46.3 & 18.8 & 41.5 & 34.6 & 4.1 & 30 & 1.8 & 177 & 130.8 \\
\hline Bare Soil & 3.7 & 30.8 & 48.7 & 3.2 & 1.7 & 2.3 & 9.6 & 100 & 69.2 \\
\hline Built-up Area & 0.7 & 1.5 & 7.5 & 3.5 & 2.9 & 0.2 & 0.5 & 16.8 & 9.4 \\
\hline Bushland & 24.5 & 38.5 & 47.2 & 72.3 & 53.7 & 59.8 & 9.7 & 305.6 & 233.4 \\
\hline Forest & 17.3 & 25.3 & 22.1 & 101.4 & 118.5 & 12.2 & 3.9 & 300.8 & 182.3 \\
\hline Grassland & 7 & 11 & 2.1 & 2.7 & 29.3 & 2.4 & 17 & 71.6 & 69.1 \\
\hline Water & 0.1 & 1.9 & 3.7 & 0.1 & 0.1 & 0.2 & 0.9 & 6.9 & 6 \\
\hline Total in year 2010 & 99.5 & 127.8 & 172.7 & 217.7 & 210.4 & 107.1 & 43.5 & 978.8 & \\
\hline Gross loss & 53.3 & 97 & 165.2 & 145.4 & 91.9 & 104.7 & 42.6 & & \\
\hline
\end{tabular}

Table 6. Land cover change by cross tabulation for GPGM for the years 2017-2027.

\begin{tabular}{cccccccccc}
\hline & \multicolumn{1}{c}{ Year: 2017 } \\
\hline Year 2027 & $\begin{array}{c}\text { Agriculture with } \\
\text { scattered settlements }\end{array}$ & $\begin{array}{c}\text { Bare } \\
\text { Soil }\end{array}$ & $\begin{array}{c}\text { Built-up } \\
\text { Area }\end{array}$ & Bushland & Forest & Grassland & Water & $\begin{array}{c}\text { Total in year } \\
\text { 2027 }\end{array}$ & $\begin{array}{c}\text { Gross gain } \\
\text { [Total-unchanged] }\end{array}$ \\
\hline $\begin{array}{c}\text { Agriculture with } \\
\text { scattered settlements }\end{array}$ & 148.2 & 4.6 & 3.7 & 14.8 & 0 & 6.7 & 0 & 177.9 & 29.7 \\
Bare Soil & 0.3 & 44.6 & 6.4 & 0.1 & 0 & 0.1 & 0 & 51.4 & 6.8 \\
Built-up Area & 0 & 0 & 5.5 & 2.6 & 4.2 & 0 & 0 & $\mathbf{1 2 . 3}$ & 6.8 \\
Bushland & 11.2 & 30.4 & 0.6 & 189.1 & 20.8 & 46.3 & 0 & $\mathbf{2 9 8 . 4}$ & 109.3 \\
Forest & 14.8 & 10.5 & 0 & 99 & 215.2 & 0 & 0 & $\mathbf{3 3 9 . 5}$ & 124.3 \\
Grassland & 2.6 & 8.8 & 0 & 0 & 60.6 & 18.5 & 4.4 & $\mathbf{9 5}$ & 76.4 \\
Water & 0 & 1.1 & 0.6 & 0 & 0 & 0 & 2.5 & $\mathbf{4 . 2}$ & 1.7 \\
Total in year 2017 & $\mathbf{1 7 7}$ & $\mathbf{1 0 0}$ & $\mathbf{1 6 . 8}$ & $\mathbf{3 0 5 . 6}$ & $\mathbf{3 0 0 . 8}$ & $\mathbf{7 1 . 6}$ & $\mathbf{6 . 9}$ & $\mathbf{9 7 8 . 7}$ & \\
Gross loss & 28.8 & 55.4 & 11.3 & 116.5 & 85.6 & 53 & 4.4 & & \\
\hline
\end{tabular}

Table 7. Land cover change by cross tabulation for GGM for the years 1997-2010.

\begin{tabular}{cccccccccc}
\hline & \multicolumn{1}{c}{ Year: 1997 } & & & \\
Year 2010 & $\begin{array}{c}\text { Agriculture with } \\
\text { scattered settlements }\end{array}$ & $\begin{array}{c}\text { Bare } \\
\text { Soil }\end{array}$ & $\begin{array}{c}\text { Built-up } \\
\text { Area }\end{array}$ & Bushland & Forest & Grassland & Water & $\begin{array}{c}\text { Total in year } \\
\text { 2010 }\end{array}$ & $\begin{array}{c}\text { Gross gain } \\
\text { [Total-unchanged] }\end{array}$ \\
\hline $\begin{array}{c}\text { Agriculture with } \\
\text { scattered settlements }\end{array}$ & 441.8 & 1.4 & 9.5 & 171.2 & 241.4 & 84.5 & 0.3 & $\mathbf{9 5 0}$ & 508.2 \\
Bare Soil & 58.1 & 2.7 & 10.1 & 346.7 & 242.6 & 259.3 & 12.1 & $\mathbf{9 3 1 . 6}$ & 928.9 \\
Built-up Area & 12 & 0.5 & 14.9 & 67.2 & 44.5 & 76.6 & 2.3 & $\mathbf{2 1 7 . 9}$ & 203 \\
Bushland & 194.9 & 2.5 & 27.1 & 3713.00 & 4385.40 & 540.9 & 1 & $\mathbf{8 8 6 4 . 8 0}$ & 5151.80 \\
Forest & 24.9 & 0 & 3.2 & 1478.80 & 4287.70 & 20.6 & 0.2 & $\mathbf{5 8 1 5 . 4 0}$ & 1527.70 \\
Grassland & 151.2 & 6.3 & 67.1 & 1154.20 & 589.7 & 918.9 & 2.3 & $\mathbf{2 8 8 9 . 6 0}$ & 1970.70 \\
Water & 4.6 & 0.3 & 2.3 & 32.9 & 36 & 34 & 1.1 & $\mathbf{1 1 1 . 2}$ & 110.1 \\
Total in year 1997 & $\mathbf{8 8 7 . 5}$ & $\mathbf{1 3 . 8}$ & $\mathbf{1 3 3 . 9}$ & $\mathbf{6 9 6 4 . 0 0}$ & $\mathbf{9 8 2 7 . 3 0}$ & $\mathbf{1 9 3 4 . 8 0}$ & $\mathbf{1 9 . 2}$ & $\mathbf{1 9 , 7 8 0 . 5 0}$ \\
Gross loss & 445.7 & 11.1 & 119.1 & 3251.00 & 5539.60 & 1015.90 & 18.1 & & \\
\hline
\end{tabular}


Table 8. Land cover change by cross tabulation for GGM for the years 2010-2017.

\begin{tabular}{|c|c|c|c|c|c|c|c|c|c|}
\hline \multicolumn{10}{|c|}{ Year: 2010} \\
\hline Year 2017 & $\begin{array}{c}\text { Agriculture with } \\
\text { scattered settlements }\end{array}$ & Bare Soil & $\begin{array}{c}\text { Built-up } \\
\text { Area }\end{array}$ & Bushland & Forest & Grassland & Water & $\begin{array}{l}\text { Total in } \\
\text { year } 2017\end{array}$ & $\begin{array}{c}\text { Gross gain } \\
\text { [Total-unchanged] }\end{array}$ \\
\hline $\begin{array}{c}\text { Agriculture with } \\
\text { scattered settlements }\end{array}$ & 102.7 & 26.4 & 22.2 & 968.6 & 199.4 & 580 & 0.2 & 1899.50 & 1796.80 \\
\hline Bare Soil & 11.5 & 570.8 & 55 & 64.7 & 36.4 & 193.3 & 69.8 & 1001.40 & 430.7 \\
\hline Built-up Area & 29.3 & 115 & 79.1 & 60.2 & 8.8 & 190.3 & 0.5 & 483.2 & 404.1 \\
\hline Bushland & 113 & 18.5 & 8.9 & 2273.50 & 1115.60 & 542.1 & 0 & 4071.60 & 1798.10 \\
\hline Forest & 395.8 & 128.7 & 39 & 3736.50 & 4030.10 & 440.8 & 3 & 8773.90 & 4743.80 \\
\hline Grassland & 296.7 & 23.7 & 6.6 & 1758.10 & 424.6 & 933.4 & 0.4 & 3443.40 & 2510.00 \\
\hline Water & 1 & 48.6 & 7.1 & 3.2 & 0.4 & 9.8 & 37.4 & 107.5 & 70.1 \\
\hline Total in year 2010 & 950 & 931.6 & 217.9 & 8864.80 & 5815.40 & 2889.60 & 111.2 & $19,780.50$ & \\
\hline Gross loss & 847.4 & 360.8 & 138.8 & 6591.30 & 1785.20 & 1956.20 & 73.8 & & \\
\hline
\end{tabular}

Table 9. Land cover change by cross tabulation for GGM for the years 2017-2027.

\begin{tabular}{|c|c|c|c|c|c|c|c|c|c|}
\hline \multirow[b]{2}{*}{ Year 2027} & \multicolumn{9}{|c|}{ Year: 2017} \\
\hline & $\begin{array}{c}\text { Agriculture with } \\
\text { scattered settlements }\end{array}$ & $\begin{array}{l}\text { Bare } \\
\text { Soil }\end{array}$ & $\begin{array}{l}\text { Built-up } \\
\text { Area }\end{array}$ & Bushland & Forest & Grassland & Water & $\begin{array}{c}\text { Total in } \\
\text { year } 2017\end{array}$ & $\begin{array}{c}\text { Gross gain } \\
\text { [Total-unchanged] }\end{array}$ \\
\hline $\begin{array}{c}\text { Agriculture with } \\
\text { scattered settlements }\end{array}$ & 1174.7 & 0.0 & 4.4 & 251.7 & 11.2 & 433.6 & 0.0 & 1875.6 & 700.9 \\
\hline Bare Soil & 0.7 & 866.6 & 16.2 & 0.4 & 1.3 & 195.9 & 20.8 & 1101.9 & 235.3 \\
\hline Built-up Area & 19.4 & 35.8 & 372.3 & 1.0 & 0.1 & 222.9 & 0.0 & 651.5 & 279.2 \\
\hline Bushland & 55.5 & 0.0 & 0.0 & 2905.8 & 935.1 & 212.5 & 0.0 & 4109.0 & 1203.1 \\
\hline Forest & 307.4 & 82.8 & 76.1 & 469.9 & 7626.2 & 44.1 & 0.1 & 8606.5 & 980.3 \\
\hline Grassland & 340.7 & 0.0 & 0.0 & 442.3 & 199.3 & 2322.1 & 0.0 & 3304.3 & 982.2 \\
\hline Water & 0.8 & 15.9 & 14.2 & 0.0 & 0.0 & 11.4 & 86.6 & 129.0 & 42.4 \\
\hline Total in year 2010 & 1899.1 & 1001.2 & 483.2 & 4071.1 & 8773.1 & 3442.6 & 107.5 & $19,777.7$ & \\
\hline Gross loss & 724.4 & 134.6 & 110.9 & 1165.2 & 1146.9 & 1120.5 & 20.9 & & \\
\hline
\end{tabular}

Table 10. Classified accuracy assessment report for GPGM.

\begin{tabular}{|c|c|c|c|c|c|}
\hline \multicolumn{6}{|c|}{ Classification Accuracy assessment report (Accuracy totals) } \\
\hline Class & Reference & Classified & Number & Producers & Users \\
\hline Name & Totals & Totals & Correct & Accuracy & Accuracy \\
\hline Agriculture & 29 & 22 & 16 & $55.20 \%$ & $72.70 \%$ \\
\hline Bare Soil & 26 & 26 & 18 & $69.20 \%$ & $69.20 \%$ \\
\hline Built-up Area & 11 & 20 & 11 & $100.00 \%$ & $55.00 \%$ \\
\hline Bushland & 27 & 24 & 19 & $70.40 \%$ & $79.20 \%$ \\
\hline Forest & 22 & 23 & 18 & $81.80 \%$ & $78.30 \%$ \\
\hline Grassland & 15 & 14 & 12 & $80.00 \%$ & $85.70 \%$ \\
\hline Water & 16 & 17 & 15 & $93.80 \%$ & $88.20 \%$ \\
\hline Totals & 146 & 146 & 109 & & \\
\hline \multicolumn{6}{|c|}{ Overall Classification Accuracy $=74.7 \%$} \\
\hline
\end{tabular}


Table 11. Kappa statistics for assessing the accuracy of GPGM forecasted land cover map 2017.

\begin{tabular}{cc}
\hline \multicolumn{1}{c}{ KAPPA (K) STATISTICS } & \\
\hline \multicolumn{1}{c}{ Overall Kappa Statistics $=70.2 \%$} & \\
\hline Conditional Kappa for each Category. & Kappa \\
\hline Land cover & 0.66 \\
\hline Agriculture & 0.868 \\
Water & 0.513 \\
Built-up Area & 0.744 \\
Bushland & 0.744 \\
Forest & 0.841 \\
Grassland & 0.626 \\
\hline
\end{tabular}

Table 12. Classified Accuracy Assessment Report for GGM.

\begin{tabular}{|c|c|c|c|c|c|}
\hline \multicolumn{6}{|c|}{ Classification Accuracy Assessment Report (Accuracy Totals) } \\
\hline Class & Reference & Classified & Number & Producers & Users \\
\hline Name & Totals & Totals & Correct & Accuracy & Accuracy \\
\hline Agriculture & 25 & 22 & 18 & $72.00 \%$ & $81.80 \%$ \\
\hline Water & 26 & 15 & 15 & $57.70 \%$ & $100.00 \%$ \\
\hline Built-up Area & 26 & 22 & 16 & $61.50 \%$ & $72.70 \%$ \\
\hline Bushland & 28 & 31 & 21 & $75.00 \%$ & $67.70 \%$ \\
\hline Forest & 43 & 58 & 39 & $90.70 \%$ & $67.20 \%$ \\
\hline Grassland & 26 & 22 & 17 & $65.40 \%$ & $77.30 \%$ \\
\hline Bare Soil & 25 & 29 & 16 & $64.00 \%$ & $55.20 \%$ \\
\hline Totals & 199 & 199 & 142 & & \\
\hline \multicolumn{6}{|c|}{ Overall Classification Accuracy $=71.4 \%$} \\
\hline
\end{tabular}

Table 13. Kappa statistics for assessing the accuracy of GGM forecasted land cover map 2017.

\begin{tabular}{cc}
\hline \multicolumn{1}{c}{ KAPPA (K) STATISTICS } & \\
\hline \multicolumn{1}{c}{ Overall Kappa Statistics $=66.1 \%$} & \\
\hline \multicolumn{1}{c}{ Conditional Kappa for each Category. } & Kappa \\
\hline Land cover & 0.7921 \\
\hline Agriculture & 1 \\
Water & 0.6863 \\
Built-up Area & 0.6246 \\
Bushland & 0.5821 \\
Forest & 0.7386 \\
Grassland & 0.4873
\end{tabular}




\subsection{Land Cover Map}

\subsubsection{Land Cover Map at GPGM}

The examination of the land cover map in 1997, showed the forest to be the dominant land cover in the study area. During that period there were no mining activities in the area. The project commenced operations in 1998. Thus, by 2010 part of the forest has been replaced by other land covers and bushland is seen as the dominant land cover, due to the fact that, re-vegetation of the degraded mining sites was done concurrently with the mining operations. The satellite image of 2017 has shown that the area which was initially covered by forest has not returned into its original state and bushland is seen to dominate the area (Figure 2). During this period the project had already ceased and the areas which were seen covered by bushland are the rehabilitated areas.

\subsubsection{Land Cover Map at GGM}

Like at GPGM, in 1997 forest was the dominant land cover type in the study area. During that period there were no mining activities in the area. The mining activities commenced in 2000. In 2010 the portion of forest was replaced by other land covers and bushland is seen as the dominant land cover scattered all over the study area. The satellite image of 2017 showed that the areas which were initially covered by forest have not yet returned to their original state yet, but are seen as the dominant land cover type (Figure 3 ).

\subsection{Change Detection}

\subsubsection{Change Detection Analysis at GPGM}

Gold Mining in GPGM resulted in widespread land cover change between 1997-2017. Data in Table 2 reveal that both positive and negative changes occurred in the land cover pattern of GPGM. For example, in 1997 before commencement of the mining operations, the largest mining licensed area was covered by forest (52.1\%), representing 510.0 ha. However, in 2010 forest area had decreased by $21.4 \%$ representing 209.0 ha and agriculture with scattered settlements decreased to $10.2 \%$ representing 100.0 ha. The analysis confirmed that, conversion of forest and agricultural land to mining pits, waste rock dumps, tailing storage facilities and other components were the most important factor leading to land cover changes within the mining area (Table 2 and Figure 4). However, in 2017, there was a slight increase in the forest area to $31.0 \%$ representing 303.0 ha and agriculture with scattered settlement to $17.7 \%$ representing 173.0 ha. The areas for forest and bushland increased from 20102017 due to progressive rehabilitation conducted in the area. It is also projected that, from 2017-2027 the areas under forest will increase from 303.0 ha (31.0\%) to 340.0 (34.7\%) (Table 2 and Figure 4). The area of forest is projected to increase, if the rehabilitated areas won't be disturbed by socio-economic activities such as small-scale mining, livestock grazing and poles collection, which involves cutting down trees. 


\subsubsection{Change Detection Analysis at GGM}

In 1997 before commencement of the mining operations the largest area of the mining licensed land was under forest (49.7\%), representing 9833.0 ha. But in 2010, the forest area had decreased to $29.3 \%$ representing 5792.0 ha. Decrease in of forest area implies that mining operations were actively conducted during this period (Table 3 and Figure 5). However, in 2017, there was considerable increase in the forest area from 5792.0 ha $(29.3 \%)$ to 8728.0 ha $(44.1 \%)$ while the area under bushland recorded significant decrease from 8878.0 ha (44.9\%) to 4093.0 ha (20.7\%). It was also projected that, from 2017-2027 forest will be the dominant land cover type, however, the area will slightly decrease from 8728.0 ha $(44.1 \%)$ to 8639.0 ha $(43.7 \%)$ (Table 3 and Figure 5). The area of forest is projected to decrease because GGM is still active hence there is a possibility of logging of trees and clear-cutting or burning of vegetation. Also, the nearby communities are using the forest resources to obtain ecosystem services such as fodder, timber, construction materials, charcoal and firewood. However, the area for bushland increased in 2010 due to progressive rehabilitation and the decrease in 2017 could be due to socio-economic activities within the area such as charcoal making, livestock grazing and trespassing for low grade rocks and Tailing Storage Facility slurry.

\subsection{Transition Probability and Future Land Cover}

\subsubsection{Transition Probability Matrix for GPGM}

Cross tabulation is a means of determining quantities of conversions from a one land cover to another. The type of change within the seven classes of LC patterns can be depicted from the change trends in the Markov transition matrices between the period 1997-2017. The probability that each LC class remained unchanged is shown by diagonal values, whereas those which changed from one class to another are presented by off-diagonal values [11]. For example, in 1997-2010 the highest unchanged transition probability was forest 119.8 ha (Table 4). This indicates that the economic activities within the area did not cause large transformation of forest to other land covers. This has also been the case for the year 2010-2017 where the highest unchanged Land Cover was forest 118.5 ha and the lowest unchanged cover was water bodies 0.9 ha (Table 5). The predicted land covers which will not be changed by the year 2027 at GPGM are forest 215.2 ha, bush land 189.1 ha, agriculture with scattered settlement 148.2 ha, bare soil 44.6 ha, built up area 5.5 ha, grassland 18.5 ha and water bodies 2.5 ha (Table 6). The results of the transition matrices for the years 1997-2010, 2010-2017 and 2017-2027 indicate clearly that, the highest unchanged transition probability is forest and the lowest unchanged transition probability is water.

\subsubsection{Transition Probability Matrix for GGM}

Tables 7-9 provide results on overall change of land cover classes (in ha) for GGM for the years 1997-2010, 2010-2017 and 2017-2027 respectively. From 1997-2010, the results on Table 7 show that the land cover areas that were un- 
changed to other land uses were agriculture 441.8 ha, bare soil 2.7 ha, built-up area 14.9 ha, bush land 3713.0 ha, forest 4288.0 ha, grassland 918.9 ha and water $1.1 \mathrm{ha}$. The results for the years 2010-2017 show that the land cover areas that were found unchanged are agriculture $102.7 \mathrm{ha}$, bare soil $570.8 \mathrm{ha}$, built-up area 79.1 ha, bush land 2273.5 ha, forest 4030.1 ha, grassland 933.4 ha and water 37.4 ha (Table 8). The predicted land covers which will not be changed by the year 2027 at GGM are agriculture with scattered settlement 1174.7 ha, bare soil 866.6 ha, built up area 372.3 ha, bush land 29.5.8 ha, forest 7626.2 ha, grassland 2322.1 ha and water bodies 86.6 ha (Table 9). The results of the transition matrices for the years 1997-2010, 2010-2017 and 2017-2027 indicate clearly that, the highest unchanged transition probability is forest and the lowest unchanged transition probability is water.

\subsubsection{Analysis of Future Trend of Land Cover Classification}

In this study, the 1997, 2010 and 2017 land cover maps were used to classify the future land cover of 2027. Future land covers can be observed in Table 3, Figure 4 for GPGM and Table 4, Figure 5 for GGM. It is seen in the projected land cover map of 2027 of GPGM that, the large part of the area is covered by forest followed by bushland then agricultural land with scattered settlements (Figure 2). These projected results may be useful references to the regulatory agencies and the mining companies in dealing with management challenges and planning for future land use. The future land cover map within the specified period was analyzed using CA-Marcov model.

In the projected land cover map of 2027 of GGM, the large part of the area is seen to be covered by forest followed by bushland then grassland (Figure 3). These projected results may be useful references to the regulatory agencies and the mining companies in dealing with management challenges and planning for future land use. Marcov chain model was also used to analyze the future land cover map within the specified period.

\subsection{Accuracy Assessment}

\subsubsection{Classification of Accuracy Assessment for GPGM}

The final stage of classification is to determine accuracy assessment. Each of the land cover map was compared to the reference data to assess the accuracy of the classified images. Accuracy Assessment classified for GPGM presented in the Classified accuracy assessment report (Table 10) indicated that the overall accuracy is $74.7 \%$ and the accuracy assessment of the forecasted land cover map to evaluate its agreement with the actual land cover using Kappa statistics was $70.2 \%$ (Table 11). These assessments indicate that there is substantial agreement between the classified land cover and actual land cover.

\subsubsection{Classification Accuracy Assessment for GGM}

Accuracy Assessment classified for GGM presented in the Classified accuracy assessment report (Table 12) indicate that the overall accuracy is $71.4 \%$ which is 
acceptable and the accuracy assessment of the forecasted land cover map to evaluate its agreement with the actual land cover using Kappa statistics was 66.1\% (Table 13) indicating almost perfect agreement.

\section{Discussion}

\subsection{Land Cover Change Detection}

The land cover changes observed in GPGM and GGM have superficial impacts on plant species in natural habitats in the past 20 years. The multi-temporal satellite data generated from the mining areas provide evidence on the detected changes in land cover types based on the development stage of the mining activities. The impacts of mining operations in the study area within the period under review indirectly influenced adjacent land uses [2]. The results from the Land Cover analysis showed that, for the year 1997 at GPGM before the project started operations in 1998, forest was found to be the dominant land cover type, covering over $50 \%$ of the total study area, followed by bushland, then followed by agriculture with scattered settlements, grassland, built up area, bare soil and the water were not detected in this period.

Our satellite-based analysis established that, between the years 1997-2010 at GPGM, the area of forest and agriculture decreased due to the mining activities in the active mining phase. At this time, a large number of trees in the forest were cut and the agricultural land was replaced by components of the mining infrastructure such as open pit, waste rock dumps, tailing storage facility, processing plant [24], infrastructure (access and energy) and construction of camps and town [30]. This is supported in the study conducted by Perpetual [16] who found that, between 1990-2010 high density forest and sparse forest in the Prestea mining area in Ghana were lost to pave way for mining operations. For the case of decreased agricultural land, this study is in line with the study conducted by [31], on the effects of loss of agricultural land due to large-scale gold mining on agriculture in Ghana. The authors confirmed that, a total of 4935.3 ha of agricultural land, representing $25.5 \%$ of the three concessions, were lost due to large-scale gold mining operations in the study period. The results are also supported in the studies conducted by [23] who found out that, there was substantial loss of forest resulting from mining activities to pave way for mining operations such as pits development. Moreover, [32] pointed out that, mining activities have disturbed biodiversity at the mining sites of Nigeria. The major focus in this study is the natural forest cover which is a major habitat for fauna.

The decrease in the area of forest and agriculture in the active mining phase results in the increase of the areas for bushland, grassland, built up area, water and bare soil. For example, the built-up area increased because of the construction of staging area that would house project personnel, equipment and the processing plant. This is in line with the study conducted by [33], who evaluated land use/cover change with temporal satellite data and information systems and 
reported that increase in the built-up area by $135.7 \%$ was largely accompanied by the loss of vegetation cover by $29.4 \%$. Moreover, the area of bushland and grassland increased due progressive rehabilitation programs conducted in the degraded mining sites such as the area for Waste Rock Dumps. The area for bare soil increased due to the infrastructure development such as access roads and energy. Finally, the presence of Tailing Storage Facility slightly increased the area for water bodies from $0.0 \%$ to $4.5 \%$ as all the slurry from the processing plant are channeled to the Tailing storage facility.

The study findings have further revealed that, in the year 2017, bushland and forest were the leading land covers in the area and accounted for 308.0 ha (31.5\%) and 303.0 ha (30.9\%) respectively, followed by agriculture with scattered settlements while the least classified was water 7.0 ha $(0.7 \%)$. The increase in the area for bushland and forest could be associated with the progressive rehabilitation of Waste Rock Dumps and Tailings Storage Facilities conducted from the period around 2003 [34]. It was the policy of GPGM mine to carry out progressive rehabilitation of all disturbed areas that are no longer required for the mining operation in accordance with final Closure objectives.

The land cover classification at GGM for the year 1997 indicated that, forest was the dominant landcover type, which occupied $49.7 \%$. This was followed by bushland, which occupied $35.2 \%$. However, the least classified Land cover was bare soil and water, which occupied $0.1 \%$ each. The analysis also indicated that, between the years 1997-2010 forest area decreased during development and active mining phases. During these phases, a huge number of trees in the forest were cut and replaced by components of the mining such as open pits, waste rock dumps, overburden and Tailing storage facilities [24], infrastructure development (access road and energy) and construction of camps within the mining area [35].

However, in the year 2010 the bush land dominated the land covering 44.9\% of the total area, followed by forest, which occupied $29.3 \%$. While, the least classified was the area covered by water which occupied $0.6 \%$. The findings revealed that, in the year 2017 at GGM, the most dominant land cover type was forest that accounted for $44.1 \%$. This was followed by bushland, grassland, agriculture, bare soil and built up area. The least classified was water covering $0.5 \%$. The forest and bushland being the dominant land cover type in 2017 could be attributed by the progressive rehabilitation which started in 2004 .

\subsection{Transition Matrices}

GPGM and GGM are among the large-scale gold mines in Tanzania, where the forest covers have been significantly changed to non-forest covers leading to superficial loss of vegetation. Before the commencement of the projects local people from the surrounding environments used the areas for anthropogenic activities such as agriculture, cattle grazing and timber harvesting. These activities were among the drivers of land cover changes in the areas [26]. After the 
projects took off these activities ceased and mining and its associated activities have been among key drivers of land cover changes experienced in the licensed areas.

In the 1997-2010 transition matrix at GPGM, large part of forest has been transformed into bushland and built up area. The main driver of forest change was clearing of vegetation for construction of roads, construction of buildings for project personnel and the development of other infrastructure. This indicates that, the large part of the forest with tree species of more than $10 \%$ crown cover and the minimum height of $3 \mathrm{~m}$ could be removed hence changed to bushland. Also, the forest cover was cleared off to pave way for construction of project infrastructures and accommodation for project personnel. At GGM during 1997-2010 and 2010-2017 periods, the main driver of forest land cover change was also bushland. This indicates that, these changes are associated with intense deforestation resulting from transforming forest to bushland.

\subsection{Land Cover Projection (Future Land Cover) for the Year 2027}

The findings have further revealed that, in both studied sites, it was projected that in the year 2027, forest will remain to be the most dominant land cover type while water bodies will be the least land cover type. Although, the forest is projected to be the most dominant land cover type in 2027, it will not reach the total area it covered in 1997. For example, at GPGM in 1997 the area covered by forest was $52.1 \%$ and, in the projection of 2027 , it will be $34.7 \%$ and at GGM the area covered by forest in 1997 was $49.7 \%$ and in the projection, it will be $43.7 \%$.

In addition, uncontrolled gold mining operations led to loss of biodiversity and ecosystem services. Notwithstanding the increased mining industry due to the government effort on promoting investment to achieve economic development of Tanzania, there should be strong enforcement of the laws to ensure that mining companies rehabilitate the degraded mining sites to the level close to its original condition. Also, it is important for the government and mining operators to enhance monitoring measures in the revegetated areas to avoid trespassing. Thus, there is a need of involving local people in the management of the rehabilitated areas.

\subsection{Accuracy Assessment for Both GPGM and GGM}

In this study, the accuracy assessment classification was done with reference to the raw satellite images. The aaccuracy assessment classified for GPGM indicated that the overall accuracy is $74.7 \%$ and the assessment of land cover model using Kappa statistics is $70.2 \%$. Whereas, the accuracy assessment classified for GGM indicated that the overall accuracy is $71.4 \%$ and Kappa statistics is $66.1 \%$. The overall accuracy values for GPGM and GGM falls within standards suggested by [36] and the Kappa statistics were interpreted as being in substantial agreement than by chance alone [8] [29] [37]. This is confirmed in the study conducted by [38] who provided land use and land cover accuracy of $82.00 \%$ 
and Kappa (K) statistics of $77.02 \%$ which is acceptable in both accuracy total and Kappa statistics. According to [29] the overall accuracy is interpreted as almost perfect agreement and Kappa Statistics accuracy is interpreted as substantial agreement. In addition, the study conducted by [39] achieved overall classification accuracies of $95.32 \%$ and $95.13 \%$ and overall kappa statistics of 0.9237 and 0.9070 respectively for the classification of 1992 and 2012 images. Moreover, the change detection approach conducted by [23] yielded a reliable change map with an overall accuracy of $83.3 \%$ and a kappa coefficient of 0.79 which is acceptable.

\section{Conclusion and Area for Further Research}

\subsection{Conclusion}

The study aimed at monitoring land cover changes of GPGM and GGM toward 1997-2017 and prediction of 2027 by using remote sensing and GIS technologies. Supervised classification method was used to delineate land cover classes. In this study, seven major land cover classes were distinguished from satellite images of 1997, 2010 and 2017 for the mining areas of GPGM and GGM to monitor mining activities over a twenty-year period. These classes include agriculture with scattered settlement, forest, bushland, grassland, bare soil, built-up areas and water bodies. The study provides land cover maps for the years 1997-2027 and the trend of land cover changes during the time period from 1997-2017 which also gave the projection of change to the year 2027. At GPGM the results from the LULC analysis showed that, in 1997 and 2027 forest was the most dominant land cover type and in 2010 and 2017 bushland was the most dominant land cover type. Whereas, at GGM the most dominant land cover type in 1997, 2017 and 2027 was forest and in 2010 was bushland. The overall trend suggests that forest area decreased from 1997 to 2010 due to clearing to pave way for mining operations such as development of pits, Waste Rock Dump and Tailing Storage Facility which resulted into significant reduction in vegetation cover. The results also imply that, mining operations were actively conducted during this period. However, the forest and bushland cover increased from 2010-2017 due to progressive rehabilitation conducted in the area. The study revealed that considerable portion of the land cover especially the forest area was converted to other land covers during the period of 1997-2010. It was also projected that in the year 2027 the area for forest and bushland in all mines will be dominant land cover types and the least land cover will be open water bodies. These results are an indication of an excellent state of the ecosystem will be found in the GPGM and GGM in the future. Thus, it is important for the government and mining operators to enhance monitoring measures in the revegetated areas to avoid encroachment. Similarly, there is a need to have an integrated forest management approach by involving the local people in Nzega and Geita Districts, who are the direct beneficiaries of the forests. The application of remote sensing and GIS technology was successful, but it is important to point out the limitations of the study; the technology was fairly expensive due to financial constraints and the 
technology required specialized skills thus frequent training was vital.

\subsection{Area for Further Research}

Remote sensing and GIS technologies used in this study serve as a reference point for further studies; the following area needs further research: Determining and quantifying the health of vegetation in the gold mining areas using Normalized Difference Vegetation Index (NDVI) by measuring the difference between near-infrared and red light.

\section{Acknowledgements}

The authors thank USGS for permission to use the satellite images through online database.

\section{Conflicts of Interest}

The authors declare no conflicts of interest regarding the publication of this paper.

\section{References}

[1] Nzunda, H.P. (2013) Impact of Mining Activities on Land Cover and Forest Stock in Mbozi District, Mbeya Region, Tanzania. Masters Dissertation, Sokoine University of Agriculture, Morogoro.

[2] Sonter, L.J., Moran, C.J., Barrett, D.J. and Soares-Filho, B.S. (2014) Processes of Land Use Change in Mining Regions. Journal of Cleaner Production, 84, 494-501. https://doi.org/10.1016/j.jclepro.2014.03.084

[3] Atayi, J., Kabo-bah, A.T. and Akpoti, K. (2016) The Effects of Large-Scale Mining on Land Use and Land Cover Changes Using Remotely Sensed Data. International Journal of Science and Nature, 7, 724-733.

[4] Avalos, A., Vilchez, F.F., Oyolsi, N. and Flores, S.M.L.M. (2018) Analysis of the Land Use and Cover Changes in the Metropolitan Area of Tepic-Xalisco (1973-2015) through Landsat Images. Sustainability, 10, 1860. https://doi.org/10.3390/su10061860

[5] Garai, D. and Allu, N.C. (2018) Land Use/Land Cover Changes in the Mining Area of Godavari Coal Fields of Southern India. The Egyptian Journal of Remote Sensing and Space Sciences, 21, 375-381. https://doi.org/10.1016/j.ejrs.2018.01.002

[6] Marina, R., Rujoiu, M. and Bogdan, M.A. (2016) Mapping Land Cover Using Remote Sensing Data and GIS Techniques: A Case Study of Prahova Subcarpathians. Procedia Environmental Sciences, 32, 244-255. https://doi.org/10.1016/j.proenv.2016.03.029

[7] Mi, J., et al. (2019) Tracking the Land Use/Land Cover Change in an Area with Underground Mining and Reforestation via Continuous Landsat Classification. Remote Sensing, 11, 17-19. https://doi.org/10.3390/rs11141719

[8] Tagoe, N.D. and Mantey, S. (2017) Mapping the Effects of Anthropogenic Activities in the Catchment of Weija Reservoir Using Remote Sensing Techniques. GJM, 17, 6-11.

[9] Borthwick, R.R. and Wang, Y. (2015) Bird Species' Responses to Post Mine Reclamation in Alabama-A Preliminary Analysis. JASMR, 4, 1-19. 
https://doi.org/10.21000/JASMR15020001

[10] Rwanga, S.S. and Ndambuki, J.M. (2017) Accuracy Assessment of Land Use/Land Cover Classification Using Remote Sensing and GIS. International Journal of Geosciences, 8, 611-622. https://doi.org/10.4236/ijg.2017.84033

[11] Awotwi, A., Anornu, G.K., Quaye-Ballard, J.A. and Annor, T. (2018) Monitoring Land Use and Land Cover Changes Due to Extensive Gold Mining, Urban Expansion, and Agriculture in the Pra River Basin of Ghana, 1986-2025. Land Degradation and Development, 29, 3331-3343. https://doi.org/10.1002/ldr.3093

[12] Rawat, J.S. (2015) Monitoring Land Use/Cover Change Using Remote Sensing and GIS Techniques: A Case Study of Hawalbagh Block, District Almora, Uttarakhand, India. Egyptian Journal of Remote Sensing and Space Sciences, 18, 77-84. https://doi.org/10.1016/j.ejrs.2015.02.002

[13] Soffianian, A. and Madanian, M. (2015) Monitoring Land Cover Changes in Isfahan Province, Iran Using Landsat Satellite Data. Environmental Monitoring and Assessment, 187, Article No. 543. https://doi.org/10.1007/s10661-015-4442-5

[14] Ahmed, B., Ahmed, R. and Zhu, X. (2013) Evaluation of Model Validation Techniques in Land Cover Dynamics. ISPRS International Journal of Geoinformatics, 2, 577-597. https://doi.org/10.3390/ijgi2030577

[15] Ghosh, S., et al. (2017) Application of Cellular Automata and Markov-Chain Model in Geospatial Environmental Modeling-A Review. Remote Sensing Applications. Society and Environment, 5, 64-77. https://doi.org/10.1016/j.rsase.2017.01.005

[16] Perpetual, P. (2015) Assessing Land Cover Change Resulting from Surface Mining Development (A Case Study of Prestea and Its Environs in the Western Region of Ghana). Master's Thesis, Geomatics Engineering, Ghana.

[17] Fitawok, M.B., Derudder, B., Minale, A.S., Van Passel, S., Adgo, E. and Nyssen, J. (2020) Modeling the Impact of Urbanization on Land-Use Change in Bahir Dar City, Ethiopia: An Integrated Cellular Automata-Markov Chain Approach. Land, 9, 115. https://doi.org/10.3390/land9040115

[18] Urban, D.L. and Wallin, D.O. (2017) Introduction to Markov Models. In: Learning Landscape Ecology, Springer, New York, 129-142. https://doi.org/10.1007/978-1-4939-6374-4_8

[19] Aneesha, S.B. and Pratap, D. (2020) Future Land Use Land Cover Scenario Simulation Using Open Source GIS for the City of Warangal, Telangana, India. Applied Geomatics.

[20] Chen, L.P., Sun, Y.J. and Saeed, S. (2015) Monitoring and Predicting Land Use and Land Cover Changes Using Remote Sensing and GIS Techniques-A Case Study of a Hilly Area, Jiangle, China. PLoS ONE, 13, e0200493. https://doi.org/10.1371/journal.pone.0200493

[21] Basommi, P.L., Guan, Q. and Cheng, D. (2025) Exploring Land Use and Land Cover Change in the Mining Areas of Wa East District, Ghana Using Satellite Imagery. Open Geosciences, 7, 618-626. https://doi.org/10.1515/geo-2015-0058

[22] Sikdar, P., Chakraborty, S., Adhya, E. and Paul, P.K. (2004) Land Use/Land Cover Changes and Groundwater Potential Zoning in and around Raniganj Coal Mining Area, Bardhaman District, West Bengal-A GIS and Remote Sensing Approach. Journal of Spatial Hydrology, 4, 1-24.

[23] Schueler, V., Kuemmerle, T. and chröder, H.S. (2011) Impacts of Surface Gold Mining on Land Use Systems in Western Ghana. Ambio, 40, 528-539. https://doi.org/10.1007/s13280-011-0141-9 
[24] Emel, J., Plisinski, J. and Rogan, J. (2014) Monitoring Geomorphic and Hydrologic Change at Mine Sites Using Satellite Imagery: The Geita Gold Mine in Tanzania. Applied Geography, 54, 243-249. https://doi.org/10.1016/j.apgeog.2014.07.009

[25] Henckel, J., Poulsen, K.H., Sharp, T. and Spora, P. (2016) Lake Victoria Goldfields. Episodes, 39, 135-154. https://doi.org/10.18814/epiiugs/2016/v39i2/95772

[26] Werema, C., Howell, K.M., Msuya, C.A. and Macha, A. (2016) Birds of Golden Pride Project Area, Nzega District, Central Tanzania: An Evaluation of Recolonization of Rehabilitated Areas. Scopus, 36, 26-36.

[27] Rizk, I. and Rashed, M. (2015) Monitoring Urban Growth and Land Use Change Detection with GIS and Remote Sensing Techniques in Daqahlia Governorate Egypt. International Journal of Sustainability Built Environment, 4, 117-124. https://doi.org/10.1016/j.ijsbe.2015.02.005

[28] Haque, M.I. and Basak, R. (2017) Land Cover Change Detection Using GIS and Remote Sensing Techniques: A Spatio-Temporal Study on Tanguar Haor, Sunamganj, Bangladesh. The Egyptian Journal of Remote Sensing and Space Sciences, 20, 251-263. https://doi.org/10.1016/j.ejrs.2016.12.003

[29] Viera, A.J. and Garrett, J.M. (2005) Understanding Interobserver Agreement: The Kappa Statistic. Family Medicine, 37, 360-363.

[30] Pallangyo, D. (2005) Environmental Impact Assessment for Mining Activities in Tanzania: Legal Analysis. Masters Dissertation in the Faculty of Law, University of KwaZulu-Natal, Pietermartzburg.

[31] Stephen, D.C., Ayensu-ntim, G., Twumasi-ankrah, A.B. and Barimah, P.T. (2015) Effects of Loss of Agricultural Land Due to Large-Scale Gold Mining on Agriculture in Ghana: The Case of the Western Region. British Journal of Research, 2, 196-221.

[32] Adesipo, A.A., Akinbiola, S., Awotoye, O.O., Salami, A.T. and Freese, D. (2020) Impact of Mining on the Floristic Association of Gold Mined Sites in Southwest Nigeria. BMC Ecology, 20, Article No. 9. https://doi.org/10.1186/s12898-020-00276-9

[33] Erener, A., Düzgün, S. and Cevdet, A. (2012) Evaluating Land Use/Cover Change with Temporal Satellite Data and Information Systems. Procedia Technology, 1, 385-389. https://doi.org/10.1016/j.protcy.2012.02.079

[34] Sinclaire, J. (2016) Status of Environment, Golden Pride Project in Isanga Village, Lusu Ward, Nzega District, Tabora Region. Resolute Tanzania Limited.

[35] Mrisho, P.D. (2005) Environmental Impact Assessment for Mining Activities in Tanzania: Legal Analysis. Dissertation Submitted in Partial Fulfillment of the Academic Requirements for the Degree of Master of Law in the Faculty of Law, University of Kwazulu-Natal, Pietermaritzburg.

[36] Monserud and Leemans, R. (1992) Comparing Global Vegetation Maps with the Kappa Statistic. Ecological Modelling, 62, 275-293.

https://doi.org/10.1016/0304-3800(92)90003-W

[37] Abubaker, H., Mohamed, A. and Abdelrahim, S. (2013) Accuracy Assessment of Land Use \& Land Cover Classification (LU/LC) "Case Study of Shomadi Area-Renk County-Upper Nile State, South Sudan”. International Journal of Scientific and Research Publication, 3, 1-2250.

[38] Tilahun, A. and Teferie, B. (2015) Accuracy Assessment of Land Use Land Cover Classification Using Google Earth. American Journal of Environmental Protection, 4, 193-198. https://doi.org/10.11648/j.ajep.20150404.14

[39] Butt, A., Shabbir, R.S., Ahmad, S. and Aziz, N. (2015) Land Use Change Mapping 
and Analysis Using Remote Sensing and GIS: A Case Study of Simly Watershed, Islamabad, Pakistan. The Egyptian Journal of Remote Sensing and Space Sciences, 18, 251-259. https://doi.org/10.1016/j.ejrs.2015.07.003 